\title{
Glottopol
}

Revue de sociolinguistique en ligne

$35 \mid 2021$

La langue à l'école, de l'institution à la classe : quelles conceptions, quelles normes, pour quels usages?

\section{Les enseignants et l'orthographe, une enquête au cycle 3}

Jacques Crinon, Georges Ferone et Hélène Font

\section{(2) OpenEdition \\ Journals}

Édition électronique

URL : https://journals.openedition.org/glottopol/298

DOI : $10.4000 /$ glottopol.298

ISSN : 1769-7425

Éditeur

Presses universitaires de Rouen et du Havre

Référence électronique

Jacques Crinon, Georges Ferone et Hélène Font, "Les enseignants et l'orthographe, une enquête au cycle 3 », Glottopol [En ligne], 35 | 2021, mis en ligne le 01 janvier 2021, consulté le 02 octobre 2021. URL : http://journals.openedition.org/glottopol/298; DOI : https://doi.org/10.4000/glottopol.298 


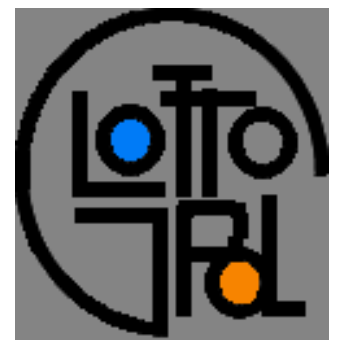

\section{GLOTTOPOL}

Revue de sociolinguistique en ligne $\mathrm{n}^{\circ} 35$ - janvier 2021

\section{La langue à l'école, de l'institution à la classe : quelles conceptions, quelles normes, pour quels usages?}

\section{SOMMAIRE}

Catherine Delarue-Breton et Élisabeth Bautier : Introduction

Catherine Delarue-Breton : Des conceptions scolaires de la langue en tension : l'exemple du programme de 2015 pour l'école primaire et le collège

Véronique Miguel-Addisu : "Sport de riche je l'ai changé en sport élitiste » : plurilinguisme et variation dans la langue de l'école du point de vue des élèves

Claire Colombel-Teuira, Véronique Fillol : Variation et approche polynomique : pour une conception plurielle de la langue à l'école calédonienne

Daphné Bloch : Pratiques langagières, situations pédagogiques et construction d'inégalités d'apprentissage à Madagascar

Samatar Abdallah Doualeh : Le français langue de l'école djiboutienne ou l'hégémonie de la norme exogène

Élise Vinel, Élisabeth Bautier : Des discours des élèves sur l'orthographe aux pratiques des enseignants, analyse d'entretiens métagraphiques

Thierry Pagnier, Belinda Lavieu-Gwozdz: Regards sur le discours scolaire: saisir des conceptions de la langue et de son enseignement

Jacques Crinon, Georges Ferone, Hélène Font : Les enseignants et l'orthographe, une enquête au cycle 3

Christel Troncy : Les enseignants, la norme scolaire et la pluralité langagière dans deux écoles immersives à programme français en Californie. Dynamiques des attitudes et des pratiques lors d'une recherche collaborative

Erwan Le Pipec: L'école, planche de salut du breton?

Argia Olçomendy : La langue basque dans les instructions officielles (1976-2019)

\section{Compte rendu de lecture}

Par Katrin Pfadenhauer: Ursula Reutner (Ed.), 2017, Manuel des francophonies, Berlin/Boston, de Gruyter, 745 p.

Par Robert Fournier: Shana Poplack, 2018, Borrowing: Loanwords in the speech community and in the grammar. New York: Oxford University Press ; xxi, $246 \mathrm{p}$.

Par Marie-Jeanne Verny: Catherine Adam, 2020, Bilinguisme scolaire. Familles, écoles, identités en Bretagne, éd. Peter Lang, coll. «Langue, multilinguisme et changement social », Berlin. 


\title{
LES ENSEIGNANTS ET L'ORTHOGRAPHE, UNE ENQUÊTE AU CYCLE 3
}

\author{
Jacques Crinon*, Georges Ferone* et Hélène Font \\ "Université Paris Est Créteil, laboratoire CIRCEFT, ESCOL
}

\section{Buts et méthodes de l'étude}

Les manières d'enseigner sont liées à des savoirs, croyances et conceptions ${ }^{1}$ qui touchent à la pédagogie, aux contenus des disciplines et aux didactiques (Borko et Putman, 1996). Ces savoirs, croyances et conceptions évoluent au cours de la vie professionnelle (Huberman, 1989) et avec la construction de 1'expérience ${ }^{2}$ (Pastré, Mayen et Vergnaud, 2006) ; ils peuvent être influencés par l'appartenance à des réseaux ou des "communautés » professionnelles, notamment aujourd'hui des communautés en ligne, au sein desquelles des enseignants, en dehors de l'institution, associent leurs classes à des projets communs, partagent des ressources, discutent de leurs pratiques (Dionne, Lemyre et Savoie-Zajc, 2010 ; Ferone et Crinon, 2020).

Dans le cadre du projet «Twictée pour apprendre l'orthographe » (TAO, programme eFRAN $^{3}$ ), nous avons conduit, en septembre-octobre 2017, auprès de 39 enseignants français du cycle $3\left(4^{\mathrm{e}}, 5^{\mathrm{e}}\right.$ et $6^{\mathrm{e}}$ années), des entretiens relatifs à leur conception de l'enseignement de l'orthographe 4 .

Une partie de ceux-ci $(n=18)$ étaient impliqués dans la Twictée, dispositif fondé sur la collaboration à distance entre enseignants, et qui consiste à échanger entre classes, via le réseau social Twitter, de courtes « dictées négociées » (Brissaud et Cogis, 2011 ; Haas, 1999) et leurs corrections argumentées. Cette pratique innovante s'est développée rapidement en quelques années et touche plusieurs centaines de classes francophones. Les dictées individuelles sont discutées en petits groupes dans la classe, puis ces dictées de groupes envoyées à une classe partenaire. Chaque erreur repérée dans les envois des partenaires fait l'objet d'un message (tweet), rédigé selon un canevas particulier indiquant la bonne orthographe, la justification de

\footnotetext{
${ }^{1}$ Nous ne distinguerons pas ici ces trois termes, aux frontières floues selon les chercheurs, qui renvoient à un ensemble imbriqué d'éléments factuels, de convictions, d'idéologies (Wanlin et Crahay, 2012).

${ }^{2}$ C'est-à-dire des leçons qu'on tire de sa pratique grâce à un retour sur celle-ci.

3 http://inspe.u-pec.fr/recherche-et-innovation/recherche-et-internationalisation/projets/tao-renforcer-lescompetences-en-orthographe-avec-le-dispositif-twictee-762893.kjsp

${ }^{4} \mathrm{Au}$ cours de cette recherche, il a également été procédé à des observations des classes de ces enseignants, à l'évaluation des progrès des élèves, à des entretiens d'élèves, au recueil d'échanges entre enseignants. Ces données ne sont pas prises en compte dans cet article. Pour d'autres analyses, voir notamment Brissaud, Viriot-Goeldel et Ponton, 2019 ; Fenoglio et Brissaud, 2020 ; Ferone et Crinon, 2021.
} 
celle-ci et un mot-clé (une « balise » dans la terminologie de Twitter) renvoyant à une typologie d'erreurs partagée 5 .

L'autre partie des enseignants interviewés $(n=21)$ enseignait l'orthographe sans cet outil. Les entretiens semi-directifs ont été conduits à partir d'une série de questions portant notamment sur la perception des compétences des élèves et des difficultés qu'ils rencontrent et sur les pratiques et manières d'enseigner l'orthographe, en lien avec les conceptions de la langue et de l'orthographe.

Ces entretiens cherchaient donc à explorer des dimensions susceptibles d'éclairer les manières de faire des répondants ${ }^{6}$. Nous leur avons demandé ce qu'ils font faire à leurs élèves et les raisons de leurs choix. Nous les avons interrogés sur les difficultés des élèves et sur l'origine qu'ils attribuent à celles-ci. Nous avons tenté de leur faire expliciter leur rapport à la langue et à l'orthographe. On peut en effet, à la suite du travail de Combaz (2017), formuler l'hypothèse d'un lien fort entre les représentations de la langue et de ses normes et les pratiques didactiques. Combaz a dégagé de grands types de rapport à la langue à travers des entretiens avec trente enseignants de cours moyen, en fonction de l'accent plus ou moins mis sur les valeurs ou sur des règles ou encore du degré d'exigence ou de tolérance. À travers quelques portraits d'enseignants, elle montre aussi la cohérence entre ces conceptions de la langue et la manière de transmettre les normes orthographiques en classe, qui n'est en outre sans doute pas sans effet sur la construction des « postures normatives » des élèves étudiées par Sautot ${ }^{7}$ (2003). Elle rejoint aussi des enquêtes auprès d'enseignants soulignant le lien entre l'insécurité vis-àvis de la langue écrite et une posture normative dogmatique (voir en particulier Péret, Sautot et Brissaud, 2008) et des recherches qui mettent en évidence la diversité des positionnements d'enseignants qui, face à un "dilemme normatif », " oscillent entre un constat d'impuissance et le choix de lutter, d'agir systématiquement » (Miguel Addisu, 2019 : 154).

Dans un article précédent (Cadet, Crinon et Ferone, 2019), nous avons présenté le contenu de ces entretiens et mis en évidence les croyances qui s'y font jour : la difficulté à appliquer les connaissances en situation gêne davantage les élèves qu'un manque de connaissances orthographiques ; maitriser l'orthographe est d'abord affaire d'entrainement ; le « raisonnement orthographique » mis en œuvre en particulier lors des corrections de dictées a d'abord pour but d'entrainer à appliquer des procédures de correction; rendre l'orthographe attrayante et motivante est un objectif essentiel... Nous avons également montré que les conceptions qui se dégagent des déclarations des «twictonautes » diffèrent peu sur ces points de celles des autres enseignants.

Cela signifie-t-il que tous les enseignants partagent complètement la même conception de l'orthographe, de son enseignement et de son apprentissage, y compris ceux qui se réclament d'une innovation qui met en avant l'argumentation orale et écrite des régularités du système ? Ou bien peut-on observer malgré tout des lignes de fracture ? C'est ce que nous cherchons ici à établir. Nous avons repris la grille d'analyse de contenu de l'étude précédente, construite selon une méthode classique d'analyse thématique (Bardin, 2013) et reproduite ci-dessous dans le tableau 1. Nous avons ensuite traité les résultats quantifiés des codages des entretiens selon la méthode statistique de l'analyse factorielle multiple (Pagès, 2002), qui permet d'établir, à partir des données elles-mêmes et sans idées préconçues, une typologie d'individus, chaque sous-

\footnotetext{
${ }^{5}$ On trouvera une description complète du dispositif et des exemples sur le site de l'association Twictée : https://www.twictee.org/twictee/

${ }^{6}$ Nous n'étudierons cependant pas dans le présent article la question complexe des liens entre les croyances et les pratiques des enseignants (voir Wanlin et Crahay, 2012) et n'utiliserons pas les observations que nous avons réalisées des classes des enseignants interrogés.

${ }^{7}$ Les postures normatives distinguées par Sautot (2003) combinent différemment des représentations linguistiques et morales de l'orthographe.
} 
population (ou « cluster ») étant caractérisée par un ensemble de réponses semblables et des occurrences moindres des réponses propres à un autre cluster.

\section{Tableau 1 : Grille d'analyse des entretiens}

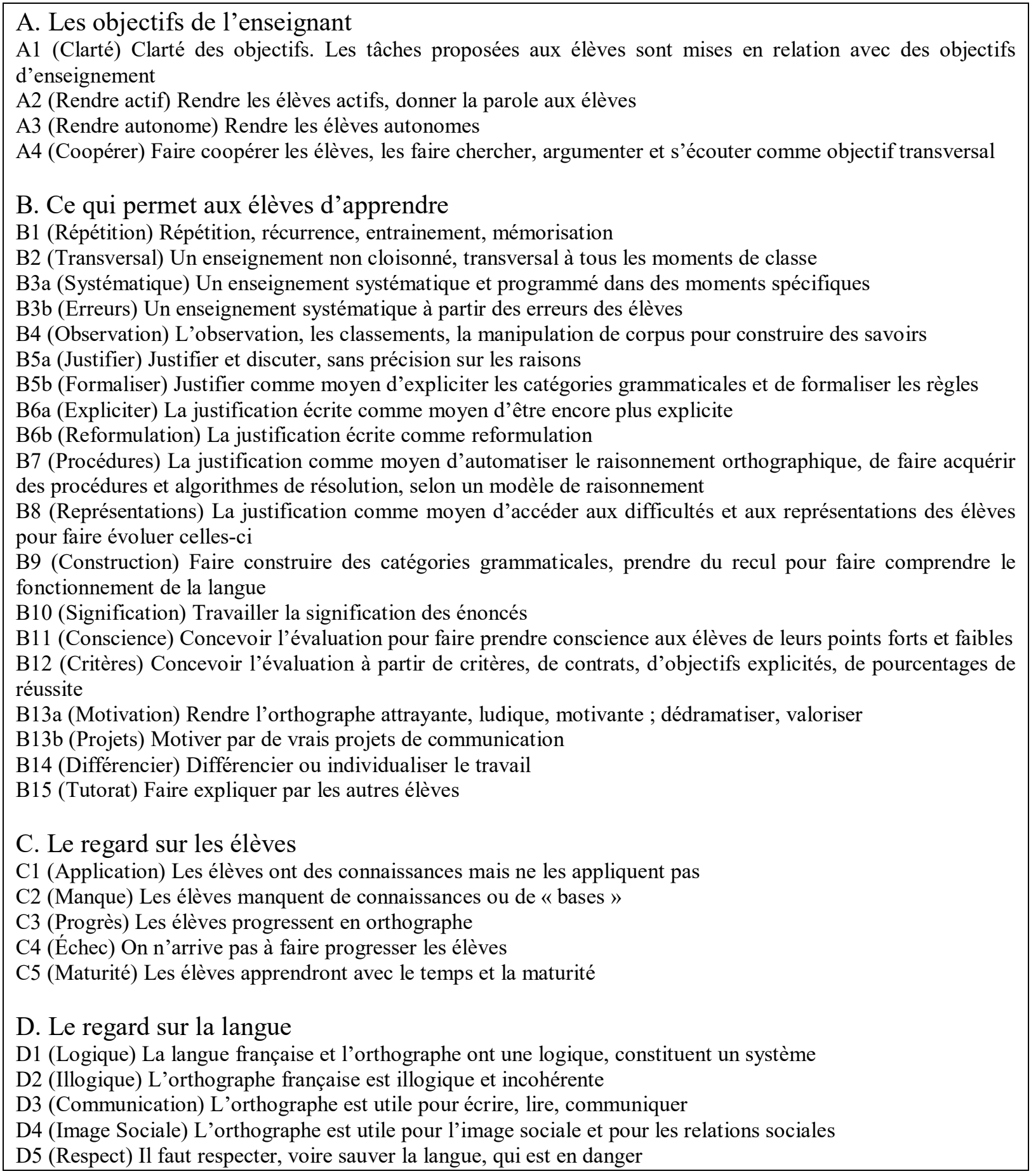




\section{Résultats}

\section{Les fréquences des catégories thématiques}

Les variables de contenu thématique ont été codées en présence / absence dans chaque entretien ${ }^{8}$. Les tableaux qui suivent présentent le nombre d'entretiens où figure chacune d'entre elles.

Tableau 2. Descriptif des variables de contenu thématique : A. Objectifs de l'enseignant

\begin{tabular}{lll}
\hline Variables & Absence N(\%) & Présence N(\%) \\
\hline A1 Clarté & $25(64.10 \%)$ & $14(35.90 \%)$ \\
A2 Rendre actif & $27(69.23 \%)$ & $12(30.77 \%)$ \\
A3 Rendre autonome & $26(66.67 \%)$ & $13(33.33 \%)$ \\
A4 Coopérer & $27(69.23 \%)$ & $12(30.77 \%)$ \\
\hline
\end{tabular}

Tableau 3. Descriptif des variables de contenu thématique : B. Ce qui permet aux élèves d'apprendre

\begin{tabular}{lll}
\hline Variables & Absence N(\%) & Présence N(\%) \\
\hline B1 Répétition & $2(5.13 \%)$ & $37(94.87 \%)$ \\
B2 Transversal & $9(23.08 \%)$ & $30(76.92 \%)$ \\
B3a Systématique & $16(41.03 \%)$ & $23(58.97 \%)$ \\
B3b Erreurs & $27(69.23 \%)$ & $12(30.77 \%)$ \\
B4 Observation & $23(58.97 \%)$ & $16(41.03 \%)$ \\
B5a Justifier & $11(28.21 \%)$ & $28(71.79 \%)$ \\
B5b Formaliser & $21(53.85 \%)$ & $18(46.15 \%)$ \\
B6a Expliciter & $30(76.92 \%)$ & $9(23.08 \%)$ \\
B6b Reformulation & $37(94.87 \%)$ & $2(5.13 \%)$ \\
B7 Procédures & $15(38.46 \%)$ & $24(61.54 \%)$ \\
B8 Représentations & $35(89.74 \%)$ & $4(10.26 \%)$ \\
B9 Construction & $27(69.23 \%)$ & $12(30.77 \%)$ \\
B10 Signification & $31(79.49 \%)$ & $8(20.51 \%)$ \\
B11 Conscience & $31(79.49 \%)$ & $8(20.51 \%)$ \\
B12 Critères & $10(25.64 \%)$ & $29(74.36 \%)$ \\
B13a Motivation & $4(10.26 \%)$ & $35(89.74 \%)$ \\
B13b Projets & $30(76.92 \%)$ & $9(23.08 \%)$ \\
B14 Différencier & $21(53.85 \%)$ & $18(46.15 \%)$ \\
B15 Tutorat & $20(51.28 \%)$ & $19(48.72 \%)$ \\
\hline & & \\
\hline
\end{tabular}

\footnotetext{
${ }^{8}$ Nous avons aussi tenté un codage « en continu », où figurait le nombre d'occurrences de chaque item de la grille d'analyse. Cette version des variables de contenu thématique n'a pas été retenue pour l'analyse factorielle multiple, car ces variables n'étaient pas normalement distribuées.
} 
Tableau 4. Descriptif des variables de contenu thématique : C. Le regard sur les élèves

\begin{tabular}{lll}
\hline Variables & Absence N(\%) & Présence N(\%) \\
\hline C1 Application & $6(15.38 \%)$ & $33(84.62 \%)$ \\
C2 Manque & $22(56.41 \%)$ & $17(43.59 \%)$ \\
C3 Progrès & $20(51.28 \%)$ & $19(48.72 \%)$ \\
C4 Échec & $32(82.05 \%)$ & $7(17.95 \%)$ \\
C5 Maturité & $29(74.36 \%)$ & $10(25.64 \%)$ \\
\hline
\end{tabular}

Tableau 5. Descriptif des variables de contenu thématique : $D$. Le regard sur la langue

\begin{tabular}{lll}
\hline Variables & Absence N(\%) & Présence N(\%) \\
\hline D1 Logique & $22(56.41 \%)$ & $17(43.59 \%)$ \\
D2 Illogique & $37(94.87 \%)$ & $2(5.13 \%)$ \\
D3 Communication & $28(71.79 \%)$ & $11(28.21 \%)$ \\
D4 Image Sociale & $25(64.1 \%)$ & $14(35.9 \%)$ \\
D5 Respect & $37(94.87 \%)$ & $2(5.13 \%)$ \\
\hline
\end{tabular}

Certaines variables seront écartées dans la suite de l'analyse, pour des raisons de technique statistique, soit parce que présentes dans plus de $90 \%$ de l'échantillon (B1), soit parce qu'absentes dans plus de $90 \%$ de l'échantillon (B6b, D2, D5). Ce déséquilibre d'effectifs aurait pu leur donner artificiellement trop de poids ${ }^{9}$.

\section{Les thèmes très fréquents et très peu fréquents}

Les fréquences de ces catégories ensuite écartées nous apportent cependant des informations qu'il convient de commenter d'abord. L'apparition de B1 dans 37 des 39 entretiens confirme la croyance massive chez les enseignants qu'apprendre à orthographier est en premier lieu une question de mémorisation et passe par la répétition et l'entrainement, certains disent même par le rabâchage, y compris en ce qui concerne l'orthographe grammaticale, qui est l'objet essentiel du dispositif Twictée. À l'opposé, n'est exprimée qu'exceptionnellement l'idée que faire formuler par écrit la justification des solutions aux problèmes orthographiques rencontrés aiderait les élèves dans la mesure où elle les oblige à reformuler avec leurs propres mots les règles et les raisonnements mis en œuvre $(\mathrm{B} 6 \mathrm{~b})$. La réflexion sur les vertus d'un passage par l'élaboration écrite - alors même que le passage par l'écrit est la première originalité de la Twictée par rapport aux dictées négociées habituelles - est peu présente.

Par ailleurs, en ce qui concerne le rapport aux normes et le regard porté sur la langue, ceux des enseignants qui abordent le sujet au cours des entretiens mettent en avant les logiques internes du système orthographique (D1), ainsi que l'utilité des compétences orthographiques pour bien communiquer par écrit (D3) ou pour éviter une stigmatisation sociale (D4).

(D1) la langue française elle a un sens et des mécaniques derrière mais elle s'explique on peut l'expliquer euh si l'enfant euh est capable de l'expliquer je pense que c'est une entrée de l'intégrer $\left(\mathrm{C} 13^{10}\right)$

\footnotetext{
${ }^{9}$ Un déséquilibre trop important rend en effet une variable moins informative dans le cadre de la création de typologies et peut nuire à la qualité des résultats.

${ }^{10}$ Les extraits d'entretiens sont référés, entre parenthèses, au numéro d'anonymat de l'enseignant interrogé.
} 
(D3) je pense que c'est vital quoi en 2017 c'est vital parce que pour tout on va devoir écrire enfin ils vont devoir écrire que ça soit sur les réseaux sociaux que ça soit pour faire des courriers voilà enfin c'est une vraie compétence sociale quoi de maitriser l'orthographe (C16)

(D4) c'est quand même un passeport euh de réussite je pense que bon bien qu'on écrive de moins en moins maintenant et qu'on écrit sur ordinateur qui corrige les fautes je trouve que c'est vraiment c'est comme le langage je trouve que ça donne vraiment un premier enfin ça permet d'avoir un premier avis sur quelqu'un (C22)

Une représentation de l'orthographe française comme faite d'incohérences et d'exceptions ou simplement d'un arbitraire qui échappe à la raison (D2) n'est qu'exceptionnellement mise en avant.

c'est dur de mettre du sens là-dessus pourquoi on écrit ça de cette façon alors que si j'écris enfant « anfan » tout le monde me comprend donc pourquoi ça s'écrit pas comme ça c'est difficile de dire aux élèves bah non ça s'écrit pas comme ça il y a quatre façons d'écrire le son [ã] donc là c'est -en c'est comme ça (C20)

Le discours sur le patrimoine menacé que constituerait l'orthographe, dont les enseignants seraient le dernier rempart, est également très rare (D5).

c'est apprendre à aimer les mots l'orthographe c'est apprendre à respecter les mots un mot s'écrit de cette manière et je le respecte en écrivant bien (C52)

je trouve que la langue française on la massacre et je supporte plus (C13)

\section{L'analyse factorielle multiple : une typologie d'enseignants}

L'analyse factorielle multiple (AFM), qui vise ici à établir une typologie des enseignants à partir des conceptions qu'ils expriment, a retenu 11 composantes principales, qui expliquent $76,5 \%$ de la variance du nuage de points. Une classification hiérarchique ascendante a ensuite été réalisée sur ces 11 composantes principales afin d'extraire deux clusters, dont la figure cidessous propose une représentation graphique dans un espace dont les axes sont les deux premières composantes de l'AFM.

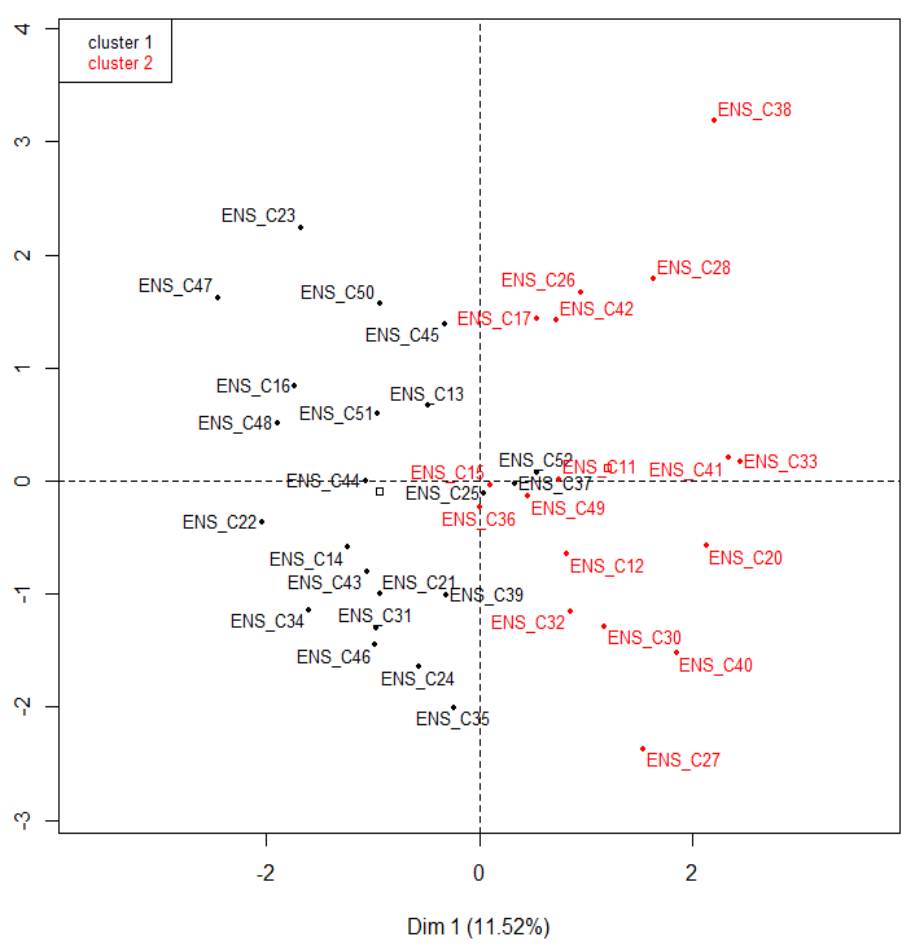

Figure 1. Représentation graphique des 2 clusters sur l'espace factoriel des 2 premières composantes de l'AFM 
Les relations entre d'une part l'appartenance au cluster 1 ou au cluster 2 et d'autre part chacune des variables de contenu thématique ont été analysées à l'aide de tests de $\mathrm{Chi}^{2}$. Il apparait que les enseignants des clusters 1 et 2 se distinguent sur 6 variables.

\section{Cluster 1 (22 enseignants)}

A4 Coopérer, chercher, argumenter et s'écouter comme objectif transversal. Aucun des enseignants du cluster $2 \mathrm{n}$ 'a mentionné la coopération entre les élèves comme étant un objectif transversal alors qu'ils sont $12(54.55 \%)$ dans le cluster 1 à avoir évoqué cet objectif lors des entretiens.

ce que je souhaite pour eux c'est qu'ils puissent vraiment avoir des échanges, même s'ils sont pas d'accord pour pouvoir trouver un consensus, qui aussi apprend des compétences sociales et civiques comment travailler ensemble (C13)

qu'est-ce qu'on gagne ? eh bien à apprendre aux enfants la collaboration (C21)

il y a de l'entraide, là il n'y a personne qui est laissé à l'écart (C48)

B14 Différencier ou individualiser le travail. Quinze enseignants $(68.18 \%)$ du cluster 1 ont mentionné la différenciation ou l'individualisation alors qu'ils sont 3 (17.65\%) dans le cluster 2 à l'avoir évoquée lors des entretiens.

voilà je différencie c'est-à-dire qu'après on va se répartir les twoutils ${ }^{11}$ à rédiger mais en fonction du niveau du groupe, je ne donnerais pas la même quantité de twoutils (C23)

j'ai aussi un système pour faire des groupes en fonction des difficultés des élèves (C44)

j'ai une pratique de classe coopérative donc avec des plans de travail avec des plans enfin un travail plus ou moins personnalisé selon les élèves et du coup qui avancent à un rythme différent au fur et à mesure de l'année donc ça veut dire qu'ils ne travaillent pas forcément les mêmes choses au même moment (C47)

C3a Les élèves progressent en orthographe. Selon 16 enseignants (72.73\%) du cluster 1, les élèves de leur classe font des progrès en orthographe, alors qu'ils ne sont que $3(17.65 \%)$ dans le cluster 2 à avoir mentionné cette croyance lors des entretiens.

je pense que oui enfin sincèrement oui moi je pense qu'ils ont plus progressé que si j'étais restée sur un dispositif enfin sur une façon d'enseigner comme avant (C14)

il y en a qui vont chercher d'autres non donc c'est j'ai vu l'évolution depuis le début de l'année déjà ça fait quatre semaines il y en a pour qui d'ailleurs très peu mettaient la marque du pluriel au début et là dans la dernière dictée j'ai vu des -ent aux verbes euh j'ai vu euh des pluriels avec des groupes nominaux accordés ; c'est pas la généralité parce qu'on est au début de l'année mais ça va venir (C21)

je suis incapable de dire si c'est que twictée qui permet ça ou si c'est l'ensemble des choses tu vois ça je peux pas en tout cas ce que je sais c'est qu'en deux ans on a énormément de progrès (C50)

\section{Cluster 2 (17 enseignants)}

B8 La justification comme moyen d'accéder aux difficultés et aux représentations des élèves pour faire évoluer celles-ci (effet tendanciel). Aucun des enseignants du cluster 1 n'a mentionné la justification de la forme choisie comme étant un moyen d'accéder aux représentations des élèves alors qu'ils sont $4(23.53 \%)$ dans le cluster 2 à avoir évoqué ce levier d'apprentissage lors des entretiens.

\footnotetext{
${ }^{11}$ Dans le dispositif Twictée, les élèves rédigent des messages (tweets) justifiant les corrections qu'ils font des dictées de leurs partenaires distants et les leur envoient : les twoutils. Ce mot-valise est formé à partir de Twitter et outil.
} 
une fois qu'on a compris ça on peut commencer à casser les représentations pour construire quelque chose de nouveau en espérant que ce soit correct (C28)

alors je leur ai demandé leur représentation, qu'est-ce que c'est que l'infinitif (C33)

c'est le secrétaire qui écrit et c'est on fait des découvertes au CM1 on s'aperçoit parfois qu'ils confondent le s et le c on s'aperçoit parfois ils veulent dire g mais ils ont dit j c'est assez intéressant ça remet les choses aussi un petit peu à leur place $(\mathrm{C} 42)$

C4 On n'arrive pas à faire progresser les élèves. Lors des entretiens, un seul enseignant $(4.55 \%)$ du cluster 1 a affirmé qu'il n'arrivait pas à faire progresser les élèves, contre 6 (35.29\%) dans le cluster 2 .

on trouve quand même qu'ils progressent peu (C33)

j'ai pas fait évoluer un enfant qui était réellement en difficulté [...] je pense que je suis pas assez compétente non plus en orthographe voilà je n'ai pas de formation suffisante certainement + oui pour pouvoir euh + peut-être que je me trompe peut-être qu'il y en a qui se sont réveillés plus tard + bon je crois pas (C38)

les élèves en difficulté ils ne voient pas de progrès c'est difficile pour eux donc je sais pas comment faire autrement (C27)

tout le monde en fait énormément [de l'orthographe] mais le résultat est quand même un manque d'efficacité (C41)

D1 La langue française et l'orthographe ont une logique, constituent un système. 4 enseignants $(18.18 \%)$ du cluster 1 ont mis en avant que la langue française et l'orthographe constituent un système alors qu'ils sont $13(76.47 \%)$ dans le cluster 2 à avoir évoqué cette représentation lors des entretiens.

l'objectif à terme c'est d'avoir en fait dans en fait d'avoir dans la classe des affichages et dans les cahiers des outils qui leur permettent de se débrouiller et de retenir quelque chose de stable on ne mémorise pas l'instable on mémorise le stable (C12)

ce que j'essaye de faire c'est d'insister sur le fait qu'en orthographe il y a beaucoup de régularités et que si on apprend des listes de mots c'est pas simplement pour apprendre dix mots par semaine mais c'est vraiment parce que ces mots-là vont s'écrire comme d'autres et donc je vais insister làdessus de ne pas chercher des orthographes farfelues (C15)

pour moi tout est là quoi, il faut observer la langue comprendre les liens et ensuite une fois qu'on a compris ça ça peut venir enfin (C33)

lier ça avec un enseignement de la langue pour rendre la langue logique parce que en fait je me suis rendu compte que les élèves qui ont du mal à orthographier c'est aussi des élèves qui [...] n'ont pas compris comment ça fonctionne donc du coup l'objectif cette année c'est de voir la langue régulièrement et de réussir à la montrer comme un système je pense qu'ils pourront mieux réussir (C49)

Nous nous sommes aussi interrogés sur les relations entre l'appartenance à l'un ou l'autre des clusters et une série de variables relatives à la carrière des enseignants, à leur pratique ou non du dispositif Twictée et aux progrès de leurs élèves. Sept variables ont ainsi été testées : le sexe, le nombre d'années d'exercice de l'enseignement, le nombre d'années de pratique en cycle 3, la pratique de fonctions de formateur d'enseignants, l'exercice en éducation prioritaire l'année de l'enquête, la pratique de la Twictée, les progrès des élèves entre le début et la fin de l'année de l'enquête ${ }^{12}$. Les relations entre d'une part l'appartenance au cluster 1 ou 2 et d'autre

\footnotetext{
${ }^{12}$ Un test (dictée et exercice de transformation) a été administré aux élèves des classes participantes au début et à la fin de l'année scolaire 2017-2018 (voir Brissaud, Viriot-Goeldel et Ponton, 2019).
} 
part chacune des variables ont été analysées à l'aide de tests de $\mathrm{Chi}^{2}$. Il apparait que les enseignants des clusters 1 et 2 se distinguent sur deux de ces variables :

- La pratique de la Twictée. Les enseignants qui composent le cluster 1 sont majoritairement ceux de la condition Twictée (59.09\%) alors que le cluster 2 ne compte que $29.41 \%$ d'enseignants de cette condition.

- Le nombre d'années d'ancienneté. Les enseignants du cluster 2 sont généralement plus jeunes dans le métier que ceux du cluster 1, puisqu'ils sont $9(52.94 \%)$ à avoir entre 2 et 11 années d'ancienneté, contre 4 (18.18\%) dans le cluster 1 .

En revanche, les enseignants des deux clusters ne se distinguent pas sur les autres variables et notamment sur les évolutions des performances des élèves (qui, soulignons-le, progressent dans toutes les classes).

\section{Conclusions}

En résumé, l'analyse factorielle multiple a permis de former deux sous-populations, deux clusters, d'importance numérique presque égale (22 vs 17 individus) et de mieux les comprendre. Nous n'avons cependant pas réussi à mettre en évidence de relation entre ces conceptions contrastées et une capacité différente à faire progresser les élèves au cours de l'année scolaire de l'enquête.

Pour les enseignants du premier cluster, l'enseignement de l'orthographe s'intègre à des pratiques qui n'ont pas seulement pour but la maitrise de l'orthographe ; elles contribuent à des objectifs transversaux, en particulier apprendre à coopérer, échanger, travailler avec d'autres ; elles procèdent d'un type de pédagogie qui met l'accent sur la différenciation ou l'individualisation, à travers par exemple des «plans de travail» individualisés ou des feedback évaluatifs. Ces enseignants témoignent d'un certain optimisme sur les effets de leur action.

Les enseignants du second cluster sont moins optimistes (ou plus exigeants et plus sensibles aux difficultés persistantes dans l'atteinte des objectifs qu'ils fixent) et mettent l'accent dans leur discours sur des considérations spécifiques à la didactique de l'orthographe : expliciter la cohérence du système orthographique, s'appuyer sur l'observation des difficultés et des représentations des élèves pour cibler leurs interventions. S'agit-il cependant d'une conviction profonde structurant leurs pratiques ou d'un discours convenu ? En effet, ils ne se distinguent pas des autres quant à leur affirmation qu'il faut surtout faire mémoriser et monter des automatismes, que le travail collectif des élèves mis en avant a pour but essentiel de retrouver les étapes obligées du « raisonnement orthographique » appris.

La seconde sous-population est plus jeune dans le métier que la première et moins présente parmi les twictonautes.

Peut-on interpréter ces résultats en termes d'évolution des conceptions des enseignants au cours de leur carrière ? Nous le ferons en tout cas en termes d'un besoin de renouvèlement ressenti par une partie des enseignants au bout d'une dizaine d'années de pratique du métier. Ces résultats nous informent particulièrement sur le profil des enseignants qui adhèrent à une innovation comme Twictée et s'y engagent. Ce sont des enseignants expérimentés, enthousiastes, convaincus de l'efficacité de leurs efforts, mus par des objectifs de collaboration avec les collègues du réseau, objectifs de collaboration qu'ils cherchent également à mettre en œuvre dans la classe. L'argument de la motivation, qui revient si souvent quand ils expliquent l'intérêt d'utiliser le dispositif Twictée pour enseigner l'orthographe, vaut autant pour euxmêmes que pour leurs élèves.

Je me suis remise en question en voyant que les règles que je leur faisais écrire avec les leçons et les suivis stricts des manuels ne fonctionnaient pas mais en tout cas c'était mais d'un ennui à toute épreuve et pour eux et pour moi (C52) 
La vision qu'ils auraient de la langue et la conviction qu'il faut faire comprendre aux élèves le fonctionnement du système linguistique et faire évoluer leurs représentations des concepts grammaticaux, en rupture avec l'enseignement d'un corpus de règles et d'exceptions, apparait rarement chez eux, contrairement au groupe d'enseignants du second cluster. L'engagement des twictonautes n'est pas à lire comme lié principalement à une critique des approches scolaires traditionnelles de l'orthographe; il semble davantage tenir à des convictions pédagogiques générales qu'aux arguments didactiques mis pourtant en avant sur le site de l'association Twictée par les promoteurs du dispositif. Cela pourrait contribuer à expliquer pourquoi ces innovateurs restent habités par les certitudes dominantes sur la nécessaire automatisation des savoir-faire en matière de gestion de l'orthographe, d'ailleurs corroborées par une partie des recherches, tant en didactique qu'en psychologie (Fayol, 2017), et sont peu sensibles à d'autres recherches qui insistent sur la construction des connaissances, l'évolution des représentations des apprenants, la clarté cognitive sur les logiques du système et les obstacles spécifiques liés à des particularités et à des secteurs précis de l'orthographe française (Cogis et Brissaud, 2019 ; David, 2006).

Il faut aussi rappeler que plusieurs catégories dégagées par l'analyse de contenu, qu'on rencontre avec une fréquence importante dans de nombreux entretiens, constituent un socle de conceptions largement partagées par les répondants de notre échantillon et donc commune aux deux clusters. Ce sont celles que nous avons décrites dans un précédent article (Cadet, Crinon et Ferone, 2019) : difficultés des élèves attribuées à une incapacité à mettre en œuvre leurs connaissances en situation; apologie d'une pédagogie de l'entrainement; mise en avant de l'objectif de conduire les élèves à construire et à automatiser des raisonnements orthographiques conçus comme des algorithmes, des suites de questions à se poser; organisation d'échanges oraux entre élèves pour faire justifier l'orthographe et parvenir à l'objectif précédent; accent mis sur la motivation des élèves et la dédramatisation de l'orthographe. On est frappé ici par le béhaviorisme sous-jacent à ces conceptions. Enseigner l'orthographe est plutôt de l'ordre du montage d'algorithmes que de la réflexion sur le système de la langue, et en particulier de la langue écrite, ou de la compréhension de son fonctionnement et de ses catégories (parties du discours, fonctions syntaxiques, notion de pluriel, etc.). De manière plus générale, il s'agit d'accomplir des tâches plus que de comprendre le monde.

En outre, la typologie d'enseignants que nous avons dégagée ne rend sans doute pas compte des conceptions de l'orthographe et de son enseignement de l'ensemble de la population concernée. Notre échantillon, si divers qu'il soit quant à l'ancienneté (nous n'avions éliminé que les débutants) et quant aux lieux d'exercice (éducation prioritaire et public plus favorisé socialement, écoles urbaines et rurales de la région parisienne et de la région de Grenoble), avait cependant un inévitable point commun : il était composé de volontaires qui, non seulement se sont prêtés à l'entretien, mais ont ouvert leur classe à l'observation des chercheurs. On peut donc penser qu'il s'agit d'un certain type d'enseignants, qui assument leurs pratiques et cherchent à avoir des retours sur celles-ci afin de les faire évoluer.

\section{Références}

BARDIN Laurence, 2013, L'analyse de contenu, Presses universitaires de France, Paris. BORKO Hilda et PUTMAN Ralph T., 1996, " Learning to teach », dans David L. Berliner et Robert C. Calfee (Eds.), Handbook of educational psychology, Mac Millan, New York, p. 673-708.

BRISSAUD Catherine et COGIS Danièle, 2011, Comment enseigner l'orthographe aujourd'hui ?, Hatier, Paris. 
BRISSAUD Catherine, VIRIOT-GOELDEL Caroline et PONTON Claude, 2019, « Enseigner et apprendre l'orthographe avec la "Twictée". Premiers résultats de l'évaluation d'un dispositif innovant d'enseignement de l'orthographe », Repères, n 60, p. 107-130.

CADET Lucile, CRINON Jacques et FERONE Georges, 2019, « Former au raisonnement orthographique. Conceptions d'enseignants du cycle $3 »$, Repères, $\mathrm{n}^{\circ}$ 60, p. 153-171.

COGIS Danièle et BRISSAUD Catherine, 2019, "À la poursuite des marques de genre... », dans Clara Mortamet (dir.), L'orthographe. Pratiques d'élèves, pratiques d'enseignants, représentations, Presses Universitaires de Rouen et du Havre, MontSaint-Aignan, p. 43-71.

COMBAZ Catherine, 2017, De la variabilité du rapport des enseignants de l'école primaire à l'orthographe appréhendée comme une norme sociale : discours et pratiques, Thèse de doctorat, Université de Cergy-Pontoise.

DAVID Jacques, 2006, «L'orthographe du français et son apprentissage, historique et perspectives ", dans Renée Honvault-Ducrocq (dir.), L'orthographe en questions, Publications des Universités de Rouen et du Havre, Mont-Saint-Aignan, p. 169-177.

DIONNE Liliane, LEMYRE François et SAVOIE-ZAJC Lorraine, 2010, « Vers une définition englobante de la communauté d'apprentissage (CA) comme dispositif de développement professionnel », Revue des sciences de l'éducation, vol. 36, n ${ }^{\circ}$, p. 25 43.

FAYOL Michel, 2017, L'acquisition de l'écrit, PUF, Paris.

FENOGLIO Prisca et BRISSAUD Catherine, 2020, "Entre tâche et activité : analyse des échanges langagiers d'élèves de cycle 3 lors de l'utilisation d'un outil didactique de correction orthographique $»$, Recherches en éducation, $\mathrm{n}^{\circ} 40$, p. 10-27. En ligne : https://journals.openedition.org/ree/427

FERONE Georges et CRINON Jacques, 2020, «Interagir à distance dans une communauté d'enseignants : effets sur les conceptions relatives au numérique et à l'enseignement de l'orthographe ». Recherches en éducation, $\mathrm{n}^{\circ} 40$, p.112-123. En ligne : https://journals.openedition.org/ree/460

FERONE Georges et CRINON Jacques, 2021, à paraitre, « Se former à distance de manière collaborative. Le cas du réseau Twictée », Médiations et médiatisations, $\mathrm{n}^{\circ}$ 5. En ligne : http://revue-mediations.teluq.ca

HAAS Ghislaine, 1999, «Les ateliers de négociation graphique : une cadre de développement des compétences métalinguistiques pour des élèves de cycle $3 »$, Repères, $\mathrm{n}^{\circ} 20, \mathrm{p} .127$ 142.

HUBERMAN Michael, 1989, «Les phases de la carrière enseignante : un essai de description et de prévision », Revue française de pédagogie, $\mathrm{n}^{\circ} 86$, p. 5-16.

MIGUEL ADDISU Véronique, 2019, «La part du sociolinguistique en didactique de l'orthographe : un enjeu pour des recherches participatives », dans Clara Mortamet (dir.), L'orthographe. Pratiques d'élèves, pratiques d'enseignants, représentations, Presses Universitaires de Rouen et du Havre, Mont-Saint-Aignan, p. 147-173.

PAGÈS J., 2002, «Analyse factorielle multiple appliquée aux variables qualitatives et aux données mixtes », Revue de Statistique Appliquée, vol. 50, n 4, p. 5-37.

PASTRÉ Pierre, MAYEN Patrick et VERGNAUD Gérard, 2006, « La didactique professionnelle », Revue française de pédagogie, $\mathrm{n}^{\circ} 154, \mathrm{p} .145-198$. En ligne: http://journals.openedition.org/rfp/157

PÉRET Claudie, SAUTOT Jean-Pierre et BRISSAUD Catherine, 2008, «Les professeurs entrant dans le métier et la norme orthographique ", dans Catherine Brissaud, JeanPierre Jaffré et Jean-Christophe Pellat (dir.), Nouvelles Recherches en orthographe, Lambert Lucas, Limoges, p. 203-214. En ligne : halshs-01269772 
SAUTOT Jean-Pierre, 2003, «Construction de la norme orthographique : quelques avatars pédagogiques », Dossiers des sciences de l'éducation, $\mathrm{n}^{\circ}$ 9, p. 109-119. En ligne : halshs-01269723

WANLIN Philippe et CRAHAY Marcel, 2012, «La pensée des enseignants pendant l'interaction en classe. Une revue de la littérature anglophone », Éducation et didactique, vol. $1, \mathrm{n}^{\circ} 6$, p. 9-46. 


\section{GLOTTOPOL}

Revue de sociolinguistique en ligne

Comité de rédaction : Michaël Abecassis (University of Oxford), Salih Akin (Université de Rouen Normandie), Sophie Babault (Université de Lille), Aude Bretegnier (Université du Mans), Claude Caitucoli, Véronique Castellotti (Université de Tours), Régine DelamotteLegrand (Université de Rouen Normandie), Alexandre Duchêne (Université de Fribourg), Valentin Feussi (Université d'Angers), Robert Fournier (Carleton University, Ottawa), Stéphanie Galligani (Université Grenoble Alpes), Médéric Gasquet-Cyrus (Université AixMarseille), Emmanuelle Huver (Université de Tours), Normand Labrie (Université de Toronto), Foued Laroussi (Université de Rouen Normandie), Benoit Leblanc (Université du Québec à Trois-Rivières), Mylène Lebon-Eyquem (Université de la Réunion), Fabienne Leconte (Université de Rouen Normandie), Gudrun Ledegen (Université de Rennes), Danièle Moore (Simon Fraser University, Vancouver), Clara Mortamet (Université Jean Monnet, Saint Etienne), Alioune Ndao (Université Cheik Anta Diop, Dakar), Isabelle Pierozak (Université de Tours), Cécile Van den Avenne (Université Sorbonne Nouvelle, Paris 3).

Rédactrice en chef : Clara Mortamet.

Directrice de publication : Fabienne Leconte.

Comité scientifique: Michelle Auzanneau (Université de Paris), Margaret Bento (Université de Paris), Jacqueline Billiez (Université de Grenobles Alpes), Philippe Blanchet (Université de Rennes), Jean-Michel Eloy (Université d'Amiens), Françoise Gadet (Université Paris Nanterre), Monica Heller (Université de Moncton), Caroline Juillard (Université de Paris), Jean-Marie Klinkenberg (Université de Liège), Marinette Matthey (Université Grenoble Alpes), Marie-Louise Moreau (Université de Mons-Hainault), Robert Nicolaï (Université Côte d'Azur), Didier de Robillard (Université de Tours), Valérie Spaëth (Université Sorbonne nouvelle), Claude Truchot (Université de Strasbourg), Daniel Véronique (Université AixMarseille).

\section{Comité de lecture pour ce numéro :}

Nathalie Auger, Michèle Auzanneau, Margaret Bento, Stéphane Bonnery, Josiane Boutet, Lucile Cadet, Danièle Cogis, Claudine Garcia-Debanc, Marc Debono Régine Delamotte, JeanFrançois De Pietro, Marie-Laure Elalouf, Valentin Feussi, Laurent Gajo, Emmanuelle Huver, Christian Lagarde, Mylène Lebon-Eyquem, Fabienne Leconte, Gudrun Ledegen, Nadia Maillard, Maira Mamede, Bruno Maurer, Clara Mortamet, Fanny Rink, Valerie Spaëth, Marielle Rispail, Françoise Ropé, Eguzki Urteaga, Cécile Van den Avenne, Daniel Véronique 